\title{
Enhanced Methylarginine Characterization by Post-Translational Modification-Specific Targeted Data Acquisition and Electron-Transfer Dissociation Mass Spectrometry
}

\author{
Gene Hart-Smith, ${ }^{1}$ Jason K. K. Low, ${ }^{1}$ Melissa A. Erce, ${ }^{1}$ Marc R. Wilkins ${ }^{1}$ \\ ${ }^{1}$ NSW Systems Biology Initiative, University of New South Wales, Sydney, New South Wales, Australia
}

\begin{abstract}
When localizing protein post-translational modifications (PTMs) using liquid-chromatography (LC)tandem mass spectrometry (MS/MS), existing implementations are limited by inefficient selection of PTM-carrying peptides for MS/MS, particularly when PTM site occupancy is sub-stoichiometric. The present contribution describes a method by which peptides carrying specific PTMs of interest-in this study, methylarginines-may be selectively targeted for MS/MS: peptide features are extracted from high mass accuracy single-stage MS data, searched against theoretical PTM-carrying peptide masses, and matching features are subjected to targeted data acquisition LC-MS/MS. Using trypsin digested Saccharomyces cerevisiae Npl3, in which evidence is presented for 18 methylarginine sites17 of which fall within a glycine-arginine-rich (GAR) domain spanning $<120$ amino acids-it is shown that this approach outperforms conventional data dependent acquisition (DDA): when applied to a complex protein mixture featuring in vivo methylated Npl3, $95 \%$ more $(P=0.030)$ methylargininecarrying peptides are selected for MS/MS than DDA, leading to an $86 \%$ increase $(P=0.044)$ in the number of methylated peptides producing Mascot ion scores $\geq 20$ following electron-transfer dissociation (ETD). Notably, significantly more low abundance arginine methylated peptides (maximum ion intensities $<6 \times 10^{4} \mathrm{cps}$ ) are selected for MS/MS using this approach relative to DDA ( $50 \%$ more in a digest of purified in vitro methylated Npl3). It is also demonstrated that relative to collision-induced dissociation (CID), ETD facilitates a $586 \%$ increase $(P=0.016)$ in average Mascot ion scores of methylarginine-carrying peptides. The present PTM-specific targeted data acquisition approach, though described using methylarginine, is applicable to any ionizable PTM of known mass.
\end{abstract}

Key words: Post-translational modification, Methylarginine, Shotgun proteomics, Electron-transfer dissociation, Saccharomyces cerevisiae, mRNA-binding protein Npl3

\section{Introduction}

$\mathrm{P}$ rotein post-translational modifications (PTMs) are crucial to our understanding of biological processes [1], and

Electronic supplementary material The online version of this article (doi:10.1007/s13361-012-0417-8) contains supplementary material, which is available to authorized users.

Correspondence to: Marc R.Wilkins; e-mail: m.wilkins@unsw.edu.au post-genomic era research has witnessed significant efforts devoted towards the development of effective PTM characterization strategies. Though phosphorylation has attracted particularly intense research attention [2,3], investigations into other classes of modifications are now growing in prominence. This has seen methylation emerge as one of the most important forms of PTM [4-8].

When characterizing a protein's PTMs, the localization of modifications to specific amino acid residues is of central concern. Over recent years, liquid chromatography (LC)-tandem 
mass spectrometry (MS/MS) has established itself as the method of choice for this purpose [9]. The most widely utilized implementation of this technique, shotgun proteomics, subjects digested proteins to LC, and eluting peptides are selected for MS/MS in an automated manner using instrument specific algorithms. When using contemporary MS instrumentation, sequence database searching, and data validation, shotgun proteomics allows for the routine characterization of thousands of peptides from complex samples [10]. Nonetheless, the technique's ability to characterize complex peptide mixtures remains far from comprehensive [11], and of particular concern, the data-dependent acquisition (DDA) approach to precursor ion selection results in substantial discrimination against low abundance ions $[10,12]$. This presents a significant issue when characterizing PTMs. Variable site occupancy can lead to PTMs being present at sub-stoichiometric levels, and even when PTM site occupancy is high, modified proteins may only be present at low abundances within the sample of interest. PTM-carrying peptides are, therefore, often excluded from MS/MS analysis during DDA, preventing their characterization.

To reduce the impact of this issue, a variety of affinity methods have been used to enrich for given PTMs. These techniques, examples of which include immobilized metal affinity chromatography (IMAC) and metal oxide affinity chromatography, have, for example, been of immense benefit to the localization of phosphorylation sites [13]. When analyzing methylation, however, only relatively weak enrichment, via immunoprecipitation using antibodies [1416], is capable of being achieved. As such, reliable selection of methylated peptide ions during DDA remains considerably problematic.

Alternatively, peptides may be selected for MS/MS using targeted approaches rather than DDA. Such approaches have been applied to the specific selection of limited numbers of low abundance peptides [11], or peptides associated with single proteins [17], within individual LC-MS/MS experiments, and have been shown to increase reproducibility in proteomic assays [18]. Importantly, Schmidt et al. developed a targeted approach aimed to provide more comprehensive coverage of detectable peptide features [19] (i.e., collections of peaks derived from the same molecular ion); this was achieved by utilizing recently developed software capable of performing peptide feature extraction from single-stage MS (MS-1) data, together with MS instrumentation capable of segmenting inclusion mass lists by LC elution time. By extracting peptide features from MS-1 data and dividing these peptide features into inclusion lists to be targeted in individual LC-MS/MS experiments, these researchers demonstrated substantial improvements in the selection of low abundance precursor ions compared to DDA experiments. Importantly, this resulted in the characterization of significantly more phosphorylation sites than DDA LC-MS/MS. More recently, Domon et al. applied these approaches to enhance their characterization of the Drosophila melanogaster phosphoproteome [20].
Though the coverage of detectable peptide features can be improved using targeted approaches, comprehensive sequencing of these features nonetheless remains difficult. As such, the comprehensive selection of PTM-carrying peptides for MS/MS remains problematic when using existing techniques. In their study, Schmidt et al. described the observation of $\sim 10,000$ peptide features from their samples, of which only $\sim 80 \%$ could be selected for sequencing across five LC-MS/MS experiments [19]. Due to limitations in mass spectrometer scan speeds, the percentages of detectable peptide features capable of being selected in a single standard LC-MS/MS experiment are even less. Given that complex protein digests can readily generate over 100,000 detectable peptide features [21], the selection of PTM-carrying peptides for MS/MS can remain highly inefficient.

Even when PTM-carrying peptides are selected for MS/MS, effective localization of the peptide's modification site(s) may be problematic if the PTM is labile when subjected to collisioninduced dissociation (CID). As a means of preferentially fragmenting peptide $\mathrm{N}-\mathrm{C}_{\alpha}$ bonds - and leaving labile PTMs largely intact - electron-transfer dissociation (ETD) may instead be applied [22-24]. Methylarginine-containing peptides, for example, generally produce MS/MS spectra dominated by ions resulting from neutral losses when subjected to CID [16, $25,26]$; recent studies have, however, demonstrated spectra rich in informative peptide backbone fragments when subjecting methylarginine-containing peptides to ETD [27, 28].

The present contribution describes a method by which peptides carrying specific PTMs of interest-in this study, methylarginines - may be selectively targeted for ETD MS/MS, significantly enhancing the ability to localize the sites of these PTMs. The method involves automatically extracting features detected in high mass accuracy MS-1 data (steps 1 and 2 of Scheme 1), searching these features against theoretically derived PTM-carrying peptide masses for the protein(s) of interest using strict mass tolerance windows (step 3 of Scheme 1), creating inclusion lists from features that generate matches (step 4 of Scheme 1), and directed sequencing of the features on the inclusion list using targeted data acquisition LC-MS/MS (step 5 of Scheme 1). The utility of this approach is demonstrated by localizing the methylarginine sites of the Saccharomyces cerevisiae mRNA-binding protein Npl3. Verification of the approach is first demonstrated on purified His-Npl3, produced recombinantly and methylated in vitro using the yeast hnRNP arginine methylatransferase (Hmt1); further verification of the approach is then demonstrated on a protein mixture featuring recombinantly produced and in vivo methylated $\mathrm{Npl} 3$. Npl3 is a known Hmt1 substrate [29-31]; its state of arginine methylation affects its function, nuclear export and protein-protein interactions [31-34]. Though high mass accuracy matrix-assisted laser desorption/ionization (MALDI)-Fourier transform-ion cyclotron resonance (FT-ICR) derived data imply the presence of numerous Npl3 methylarginine sites within the protein's glycine-arginine-rich (GAR) domain [32], these sites have not as yet been verified using MS/MS. 


\section{STEP 1: PRELIMINARY LC-MS ANALYSES}

Subject the protein digest sample to preliminary high mass accuracy LC-MS analyses (a minimum of one such analysis).

Conventional shotgun-proteomics may identity the protein (or proteins) in the sample if they are not already known.

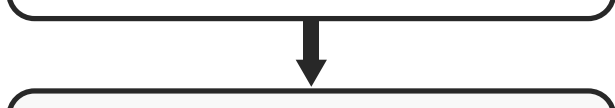

STEP 2: FEATURE EXTRACTION

Extract peptide features from the LC-MS experiment(s) of step 1 using feature extraction software.

Various different software packages may be employed (see Ref. 47). MaxQuant is utilized in the present study.

\section{STEP 3: IDENTIFY PUTATIVE PTM-CARRYING PEPTIDES}

Search the high accuracy monoisotopic masses associated with the features extracted during step 2 against theoretically derived PTM-carrying peptide masses for the protein(s) and PTM(s) of interest.

In the present study, the FindMod utility is utilized during this step.

\section{STEP 4: INCLUSION LIST GENERATION}

Extract $\mathrm{m} / \mathrm{z}$ and LC retention time data for monoisotopic masses returning matches following step 3 , and incorporate this data into an LC-MS/MS inclusion list.

For the present study, data are extracted from MaxQuant outputs in-house.

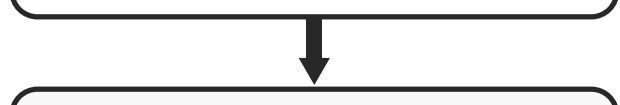

STEP 5: TARGETED LC-MS/MS

Selectively target the ions incorporated into the inclusion list of step 4 for MS/MS using targeted data acquisition.

The present study uses an inclusion list segmented by $L C$ retention time on an $L T Q$ mass spectrometer. Ions are selectively targeted for both ETD and CID MS/MS.

Scheme 1. The workflow utilized for PTM-specific targeted data acquisition LC-MS/MS

\section{Experimental}

\author{
Overexpression and In Vitro Methylation \\ of His-Npl3
}

In-depth experimental details for the overexpression, purification and in vitro methylation of His-Npl3 are included in the Supplementary Material. Briefly, Saccharomyces cerevisiae strain BY4741 (MATa his $3 \Delta 1$ leu $2 \Delta 0$ ura $3 \Delta 0$ met15 $\Delta 0$; Open Biosystems, Latayette, Colorado, USA.) $h m t 1$ and $n p l 3$ genes were amplified by PCR, and His-Hmt 1 and His-Npl3 recombinant proteins were expressed in Escherichia coli BL21DE3 Rosetta. His-tagged proteins were purified using a $\mathrm{Ni}^{2+}$-charged sepharose column (GE Healthcare, Uppsala, Uppsala, Sweden). His-Np13 was methylated in vitro using the His-Hmtl enzyme with both $S$-adenosyl- $L$-methionine (SAM) and $\left[{ }^{3} \mathrm{H}\right]-\mathrm{SAM}$; radiolabeled products were subjected to fluorography to support the efficacy of in vitro methylation. Excised polyacrylamide gel bands containing either SAM methylated or non-methylated His-Npl3 were destained, reduced and alkylated following the procedure described by Shevchenko et al. [35], and digested using trypsin (Promega, Madison, Wisconsin, USA). Digest solutions were prepared for MS in $1 \%$ formic acid and $0.05 \%$ heptafluorobutyric acid (HFBA).

\section{Overexpression and In Vivo Methylation of Npl3}

The yeast overexpression plasmid for $\mathrm{Npl3}$ was obtained from the Movable ORF (MORF) library (Open Biosystems). During verification of the plasmid via sequencing, it was found to carry a $1015 \mathrm{G}>\mathrm{T}$ point mutation. As the point mutation led to a G339X nonsense mutation in the resulting polypeptide, it was rectified through site-direct mutagenesis, in depth experimental details of which are provided in the Supplementary Material. Protease C and protein A Cterminal fusion tags were removed through the use of sitedirected ligase independent mutagenesis (SLIM) as described by Chiu et al. [36] using the primers shown in Table S-1. The plasmids were then transformed into wildtype yeast cells using the LiAc method [37] and proteins were overexpressed as described by Gelperin et al. [38] and Mok et al. [39]. Five $\mathrm{mL}$ of cells were harvested, resuspended in $1 \mathrm{~mL}$ of induction media and methylated in vivo by incubating in $50 \mu \mathrm{M}$ SAM for $90 \mathrm{~min}$. Following in vivo methylation, the cells were harvested and stored at $80{ }^{\circ} \mathrm{C}$. Prior to mass spectrometric analyses, proteins were subjected to SDS-PAGE, excised from gel bands, and digest solutions were prepared as per the experimental procedures described for the in vitro methylated His-Np13 samples.

\section{Mass Spectrometry}

Proteolytic peptides were separated by nano-LC using an UltiMate 3000 HPLC and autosampler system (Dionex, Amsterdam, The Netherlands), and ionized using positive ion mode electrospray following experimental procedures de- 
scribed previously [40]. MS and MS/MS were performed using an LTQ Orbitrap Velos (Thermo Electron, Bremen, Germany) hybrid linear ion trap and Orbitrap mass spectrometer. DDA mode and CID conditions have been described previously [40]. ETD was performed with a 130 ms reaction time, supplemental activation employed and at a target value of 10,000 ions; target values for fluoranthene anions ranged from $7 \times 10^{5}$ to $4 \times 10^{6}$. For both CID and ETD, product ions were mass analyzed in the linear ion trap.

\section{Inclusion List Generation}

MS-1 data was firstly obtained by subjecting protein digest mixtures to at least one preliminary LC-MS/MS experiment, followed by feature extraction using the MaxQuant software package (ver. 1.1.1.36; Max Planck Institute of Biochemistry, Martinsried, Germany) using standard criteria [41]. The "match between runs" feature was enabled if two or more preliminary LC-MS/MS experiments were conducted, and a time window of 2 min was used. Monoisotopic masses for extracted features (between 8500 to 23,000 in total for the samples of interest) were then run through the FindMod utility [42] (developed by us and maintained by the Swiss Institute of Bioinformatics) against the unprocessed precursor of Npl3 (accession Q01560). For the in vitro methylated Npl3 samples, a maximum number of 2 PTMs within one peptide and two missed tryptic cleavages were allowed for. For the in vivo methylated $\mathrm{Npl3}$ sample, a maximum number of 3 PTMs within one peptide and four missed tryptic cleavages were allowed for. For all samples, a mass tolerance of $\pm 2 \mathrm{ppm}$ was specified. All monoisotopic masses returning matches for peptides containing one, two, or three mono- or di-methylarginine units (in addition to any oxidized methionine and carboxyamidomethyl cysteine units) were pooled. Retention times and $\mathrm{m} / \mathrm{z}$ values of features corresponding to the pooled monoisotopic masses were extracted from the MaxQuant outputs in-house, and singly charged features removed. Retention time windows of $\pm 2 \mathrm{~min}$ were determined, and final $\mathrm{m} / \mathrm{z}$ and retention time information were inputted into an LTQ Orbitrap Velos method file as an LC elution time segmented inclusion list.

\section{Targeted MS/MS of Putatively Methylated Peptide Ions}

For targeted LC-MS/MS experiments, LC, nanospray and survey scan conditions were identical to those employed during DDA LC-MS/MS. Up to the 10 most abundant ions (>3000 counts) within the inclusion list were sequentially isolated and fragmented via CID or ETD. MS/MS conditions were as described above, with the following exception: two microscans were enabled per MS/MS experiment at target values of 50,000 ions. Inclusion list mass tolerances were set to $\pm 10 \mathrm{ppm}$, monoisotopic precursor ion selection was disabled, and $\mathrm{m} / \mathrm{z}$ values selected for MS/MS were dynamically excluded for $10 \mathrm{~s}$.

\section{Database Searches}

LC-MS/MS derived peak lists were submitted to the database search program Mascot (ver. 2.3; Matrix Science, London, UK). The following search parameters were employed: instrument type was set as ESI-TRAP or ETDTRAP for CID and ETD derived data, respectively; precursor and MS/MS tolerances were $\pm 4 \mathrm{ppm}$ and $\pm 0.4 \mathrm{Da}$, respectively; acrylamide (C), carbamidomethyl $(\mathrm{C})$, oxidation $(\mathrm{M})$, methylation $(\mathrm{R})$, and dimethylation $(\mathrm{R})$ were specified as variable modifications; enzyme specificity was trypsin with two or four missed cleavages allowed; and the SwissProt database was searched.

\section{Results}

To verify the utility of the present approach, three replicate samples of methylated His-Npl3 were analyzed using both DDA and targeted data acquisition LC-MS/MS; each precursor ion selection method was applied using both CID and ETD. The following sections describe, first, the methylated peptides confidently identified across these experiments, and the Npl3 methylarginine sites capable of being localized from these peptides. Second, comparisons of the efficacies of CID and ETD, and DDA and targeted data acquisition in characterizing these peptides are presented. Following these analyses of purified in vitro methylated HisNpl3, experiments confirming the utility of the present approach on a protein digest mixture containing in vivo methylated Npl3 are presented.

\section{Npl3-Derived In Vitro Methylated Peptide Identifications}

Cloning, expression, and purification of both His-Npl3 and His-Hmt1 were successful, as evidenced by the Coomassie stained gel of Figure S-1a. Previous research indicates that purified His-Hmt1 is functional in vitro [34], and fluorographic analysis confirmed that methylation of His-Npl3 was indeed successful (see Figure S-1b). Additional evidence for the successful methylation of His-Npl3 is observed in Figure S-1a: methylation of His-Npl3 leads to a slight retardation in gel mobility [43], as observed in lane 3.

In combination, the data generated from the various LCMS/MS experiments conducted upon the three replicate samples of methylated His-Np13 allow up to 84 methylated peptide ions to be identified. For the purposes of the present investigation, in order to allow the efficacies of the different LC-MS/MS methods to be comprehensively compared, methylated peptide ion identifications have been placed into one of three classes: "very high confidence," "high confidence," and "medium confidence." The criteria used to assign precursor ions to these different classes are described in detail in the Supplementary Material. Briefly, for a precursor ion to be identified as a very high confidence methylated peptide: at least one LC-MS/MS analysis of the precursor ion must produce a 
methylated peptide match as the top scoring Mascot hit at $P<$ 0.05 (corresponding to Mascot ion score thresholds of 36 to 38 for the present searches), and manual inspection of the peptide backbone fragment ion series must confirm the localization of the site(s) of methylation. (Observations of neutral loss-derived product ions characteristic of the peptide's identified methylation type (mono- or di-) are used as further support for precursor ion identifications.) Precursor ions that do not fulfill the abovementioned criteria may still be classified as high or medium confidence methylated peptide ions based upon: their Mascot ion score; whether their methylated peptide residue(s), as identified by Mascot, have been confirmed in other peptides; whether different charge states of the peptide are capable of being confidently identified; and the presence of neutral losses or product ions in MS/MS spectra that are characteristic of their identified methylation type(s).

Table 1 lists the highest confidence peptide identifications obtained for the different methylated and putatively methylated arginine residues observed across all LC-MS/MS analyses of the three methylated His-Npl3 samples; a complete list of the methylated peptide ions identified using LC-MS/MS may be found in Table S-2. As summarized in Table S-2, 54 precursor ions can be identified as methylated peptides with very high confidence, 25 with high confidence, and 5 with medium confidence. These peptide identifications reveal dense methylation of His-Npl3: 18 methylarginine residues may be localized (summarized in Scheme S-1), and 17 of these residues occur on Arg-Gly-Gly (RGG) or RG motif arginines within a GAR domain spanning $<120$ amino acids. Each methylated residue is identified in both unmodified and (mono- and/or di-) methylated forms, indicating the sub-stoichiometric nature of the observed methylation. Further discussions on how these findings relate to previous research conducted upon $\mathrm{Npl3}$, and on the amino acid motifs associated with Hmt1 catalyzed arginine methylation, are presented in the Supplementary Material.

\section{CID versus ETD}

To effectively localize methylarginine sites via LC-MS/MS and sequence database searching, peptide backbone fragments must be reliably observed from methylated peptide ions after they have been selected for MS/MS. CID of the abovementioned methylated $\mathrm{Npl3}$ peptide ions produce spectra dominated by neutral loss-derived fragments, which result in the observation of few and low abundance peptide

Table 1. The Highest Confidence Peptide Identifications Associated with Each Methylated and Putatively Methylated Arginine Residue Identified Across LC-MS/ MS Analyses of Three Replicate Samples of In Vitro Methylated His-Np13, Each Digested Using Trypsin. Mono-Methylated Arginine Residues are Marked with an Asterisk, and Di-Methylated Arginine Residues are Marked with Two Asterisks. All Mascot Ion Scores were Obtained from ETD Derived MS/MS Spectra. The Criteria Used to Classify the Confidence of Identifications are Outlined in the Supplementary Material, and the Superscript Roman Numerals refer to the Specific Classification Criteria used. Methylation-Specific Neutral Losses Observed in the Spectra Associated with the Listed Mascot Ion Scores are Given, and are Abbreviated as Follows: mono-methylamine (MMA), mono-methylguanidine (MMG), asymmetric di-methylamine (aDMA), and di-methylguanidine (DMG)

\begin{tabular}{|c|c|c|c|c|c|c|}
\hline $\begin{array}{l}\text { Methylated residue } \\
\text { (amino acid \#) }\end{array}$ & $\begin{array}{l}\text { Methylation type } \\
\text { (mono- or di-) }\end{array}$ & $\begin{array}{l}\text { Peptide } \\
\text { sequence }\end{array}$ & $\begin{array}{l}\text { Charge } \\
\text { state }\end{array}$ & $\begin{array}{l}\text { Mascot } \\
\text { ion score }\end{array}$ & $\begin{array}{l}\text { Confidence } \\
\text { of identification }\end{array}$ & $\begin{array}{l}\text { Methylation-specific } \\
\text { neutral losses }\end{array}$ \\
\hline \multirow[t]{2}{*}{284} & mono- & R.SNR*GGFR & $2+$ & 22 & $\operatorname{high}^{\mathrm{iv}, \mathrm{v}}$ & MMA, MMG \\
\hline & di- & R.SNR **GGFR & $2+$ & 21 & high $^{\text {iv, }}$ v & aDMA, DMG \\
\hline 288 & mono- & R.SNR**GGFR* & $2+$ & 14 & medium $^{\text {viii }}$ & MMA, MMG, aDMA, DMG \\
\hline \multirow[t]{2}{*}{290} & mono- & R.GR*GGFR*GGFR & $3+$ & 54 & very high ${ }^{\mathrm{i}}$ & MMA, MMG \\
\hline & di- & R.GR $* * G G F R * * G G F R$ & $2+$ & 3 & medium $^{\text {viii }}$ & aDMA, DMG \\
\hline \multirow[t]{2}{*}{294} & mono- & R.GR $*$ GGFR $*$ GGFR & $3+$ & 54 & very high ${ }^{\mathrm{i}}$ & MMA, MMG \\
\hline & di- & R.GGFR $* *$ GGFR & $2+$ & 22 & high $^{\text {iv, v }}$ & aDMA, DMG \\
\hline \multirow[t]{2}{*}{298} & mono- & R.GGFR*GGFR*GGFR & $3+$ & 23 & high $^{\text {iv, }}$ v & MMA, MMG \\
\hline & di- & R.GGFR $* *$ GGFR & $2+$ & 22 & $\operatorname{high}^{\text {iv, }}$ v & aDMA, DMG \\
\hline 302 & mono- & R.GGFR*GGFSR & $2+$ & 37 & very high ${ }^{\mathrm{i}}$ & MMA, MMG \\
\hline \multirow[t]{2}{*}{307} & mono- & R.GGFSR*GGFGGPR*GGFGGPR & $3+$ & 55 & very high ${ }^{\mathrm{i}}$ & MMA, MMG \\
\hline & di- & R.GGFSR $* *$ GGFGGPR $* *$ GGFGGPR & $3+$ & 87 & very high ${ }^{\mathrm{i}}$ & aDMA, DMG \\
\hline \multirow[t]{2}{*}{314} & mono- & R.GGFSR *GGFGGPR*GGFGGPR & $3+$ & 55 & very high ${ }^{\mathrm{i}}$ & MMA, MMG \\
\hline & di- & R.GGFSR $* *$ GGFGGPR $* *$ GGFGGPR & $3+$ & 87 & very high ${ }^{\mathrm{i}}$ & aDMA, DMG \\
\hline \multirow[t]{2}{*}{321} & mono- & R.GGFGGPR *GGYGGYSR & $3+$ & 61 & very high ${ }^{\mathrm{i}}$ & MMA, MMG \\
\hline & di- & R.GGFGGPR**GGYGGYSR & $3+$ & 33 & $\operatorname{high}^{\text {iv, v }}$ & aDMA \\
\hline \multirow[t]{2}{*}{329} & mono- & R.GGYGGYSR*GGYGGYSR & $3+$ & 80 & very high ${ }^{\mathrm{i}}$ & MMA, MMG \\
\hline & di- & R.GGYGGYSR **GGYGGYSR**GGYGGSR & $3+$ & 55 & very high ${ }^{\mathrm{i}}$ & aDMA, DMG \\
\hline \multirow[t]{2}{*}{337} & mono- & R.GGYGGYSR *GGYGGSR & $3+$ & 88 & very high ${ }^{\mathrm{i}}$ & MMA, MMG \\
\hline & di- & R.GGYGGYSR **GGYGGSR & $3+$ & 75 & very high ${ }^{\mathrm{i}}$ & aDMA, DMG \\
\hline 351 & mono- & R.GGYDSPR*GGYDSPR & $2+$ & 58 & very high ${ }^{\mathrm{i}}$ & MMA, MMG \\
\hline \multirow[t]{2}{*}{358} & mono- & R.GGYDSPR*GGYSR**GGYGGPR & $3+$ & 73 & very high ${ }^{\mathrm{i}}$ & MMA, MMG, aDMA, DMG \\
\hline & di- & R.GGYDSPR **GGYSR **GGYGGPR & $3+$ & 78 & very high ${ }^{\mathrm{i}}$ & aDMA, DMG \\
\hline \multirow[t]{2}{*}{363} & mono- & R.GGYDSPR*GGYSR*GGYGGPR & $3+$ & 51 & very high ${ }^{\mathrm{i}}$ & MMA, MMG \\
\hline & di- & R.GGYDSPR**GGYSR **GGYGGPR & $3+$ & 78 & very high ${ }^{\mathrm{i}}$ & aDMA, DMG \\
\hline \multirow[t]{2}{*}{377} & mono- & R.NDYGPPR*GSYGGSR**GGYDGPR & $3+$ & 64 & very high ${ }^{\mathrm{i}}$ & MMA, MMG \\
\hline & di- & R.NDYGPPR $* *$ GSYGGSR $* *$ GGYDGPR & $4+$ & 53 & very high ${ }^{\mathrm{i}}$ & aDMA, DMG \\
\hline \multirow[t]{2}{*}{384} & mono- & R.NDYGPPR*GSYGGSR*GGYDGPR & $3+$ & 64 & very high $^{\mathrm{i}}$ & MMA, MMG \\
\hline & di- & R.NDYGPPR*GSYGGSR**GGYDGPR & $3+$ & 64 & very high ${ }^{\mathrm{i}}$ & MMA, MMG, aDMA, DMG \\
\hline 391 & di- & R.GSYGGSR $* *$ GGYDGPR $* *$ GDYGPPR & $3+$ & 54 & very high ${ }^{\mathrm{i}}$ & aDMA, DMG \\
\hline 404 & mono- & R.TR*DAPR & $2+$ & 26 & $\operatorname{high}^{\text {iv, }} \mathrm{v}$ & MMA, MMG \\
\hline
\end{tabular}


backbone fragments ( $b$ and $y$ ions). This may, for example, be seen in the illustrative example provided in Figure 1a, which shows a CID spectrum obtained from the doubly charged GGYSR(dimeth.)GGYGGPR peptide (ion ID\# 65 of Table S-2). The observations of abundant neutral lossderived fragments are consistent with previous studies conducted upon methylarginine-carrying peptides [16, 25, 26]; the present experiments, however, reveal spectra that are generally dominated by ions resulting from $-\mathrm{H}_{2} \mathrm{O}$ losses, rather than the methylamine, methylguanidine, or methylcarbodiimide losses emphasized in these previous studies. Though product ions derived from these methylationspecific neutral losses are also frequently observed in the present spectra (examples may be found in Figure 1a and Figure S-2a), they are often produced at low relative abundances; in spectra such as Figure 1a, this can lead to these neutral loss-derived product ions being unable to be identified above respectable signal-to-noise thresholds.

In contrast, ETD of the same methylated Npl3 peptide ions reliably produce more and higher abundance peptide backbone fragments ( $c^{\prime}$ and $z^{\prime}$ ions) than CID; illustrative examples of this are presented in Figure $1 b$ and Figure S-2b. Methylarginine-associated neutral losses from charge-reduced precursor ions, which have previously been reported by Snijders et al. [28], are also reliably observed during ETD, the resulting product ions of which are consistently detected at high signal-to-noise ratios. This readily allows the methylation type on di-methylarginine residues (asymmetric or symmetric) to be determined [25, 26, 44], and for methylated peptide ions to be identified with confidence, even for peptides that produce poor sequence database search scores. This can be seen in Figure S-3, which shows

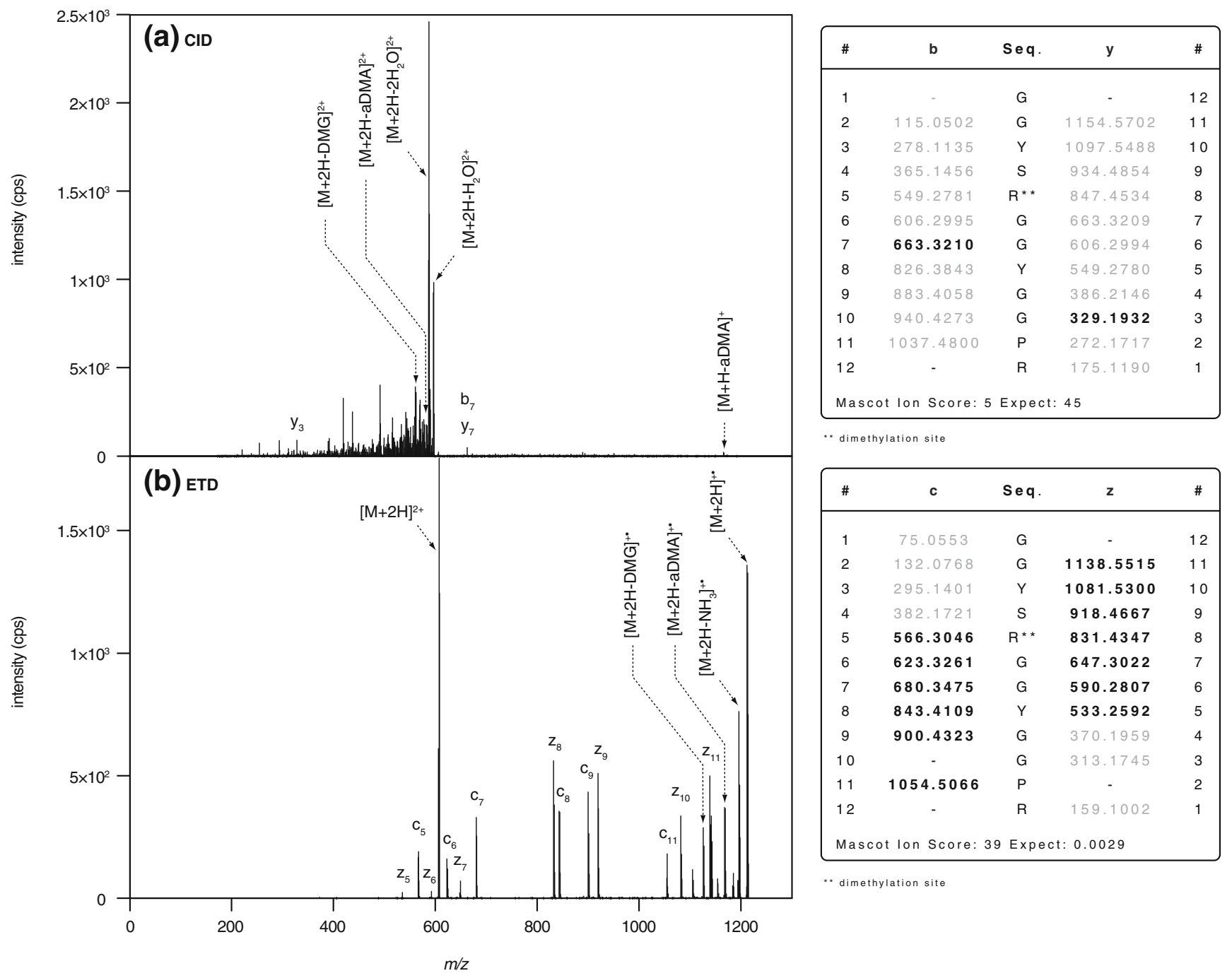

Figure 1. MS/MS spectra obtained from the doubly charged tryptic Npl3 peptide GGYSR(di-meth.)GGYGGPR (Ion ID\# 65 of Table S-2) via (a) CID, and (b) ETD. All singly charged $b$ and $y$ ions are labeled in spectrum (a), and all singly charged $c^{\prime}$ and $z^{\mathbf{1}^{\circ}}$ ions are labeled in spectrum (b). All precursor or charge-reduced precursor ions, and product ions derived from neutral losses of $-\mathrm{H}_{2} \mathrm{O},-\mathrm{NH}_{3}$, or methylation specific fragments are labeled in each spectrum. Exact masses for peptide backbone fragments are listed in the tables to the right of the spectra, and observed fragments are shown in black and bolded 
an ETD spectrum obtained from the doubly charged GGFGGPR(dimeth.)GGYGGYSR peptide (ion ID\# 32 of Table S-2) fragmented at a low ion count. Though sequence database searching of this spectrum resulted in a Mascot ion score of only 9 (as the low abundances of the product ions resulted in high mass measurement errors), the methylationspecific neutral losses of di-methylguanidine and asymmetric di-methylamine are clearly apparent, allowing the identity of this precursor ion to be confirmed with high confidence.

Compared with CID, the consistently enhanced peptide backbone fragmentation obtained via ETD for the methylated Npl3 peptide ions produce significantly improved sequence database search results. Figure 2 shows histograms illustrating the Mascot ion score distributions of methylated peptides selected for MS/MS across six DDA LC-MS/MS experiments: three using CID, and three using ETD. The Mascot ion scores are associated with precursor ions that correspond to the very high, high, and medium confidence methylated peptide ions listed in Table S-2; if only very high confidence methylated peptide ions are considered, qualitatively comparable results are obtained (data not shown). To ensure that differences in ion abundances or charge states do not introduce biases in the illustrated ion scores, the data are taken from sets of ETD and CID LC-MS/MS experiments matched in terms of the ion counts and the charge states of the methylated peptide ions (see Figure S-4). From Figure 2, it can be seen that compared with CID, the use of ETD results in a distribution skewed towards relatively high Mascot ion scores; this results in a $586 \%$ increase in average ion scores per ETD experiment relative to CID (this difference is statistically significant; $P=0.016$ from a 2tailed $t$-test). ETD is known to produce more efficient fragmentation of peptides at charge states higher than $2+$ $[22,45]$, and peptide mass has also been shown to be capable of influencing sequence database search score distributions [46]. The current data indicate that ETD outperforms CID across all observed methylated peptide charge states and masses (see Figures S-5 and S-6, which show the data of Figure 2 segmented by peptide charge states and masses). Furthermore, when ETD- and CIDderived ion scores are directly compared for each individual methylated peptide ion, in all instances bar one, ETD outperforms CID when precursor ions are fragmented at comparable ion counts (the one exception being Ion ID\# 62 of Table S-2; in the six experiments used to produce Figure 2, this doubly charged ion produced a maximum ETD-derived ion score of 20 when detected at $7.7 \times 10^{4} \mathrm{cps}$, and a maximum CID-derived ion score of 21 when detected at $7.9 \times 10^{4} \mathrm{cps}$ ). These results strongly support the notion that the use of ETD over CID consistently enhances the ability to localize methylarginine sites via sequence database searching, and that this holds true across tryptic peptides of varying charge states $(2+, 3+$, and $4+)$ and masses (700-2300 Da).

\section{Data Dependent Acquisition versus Targeted Data Acquisition}

The results discussed above indicate that, once methylated peptide ions are selected for MS/MS, ETD is the preferred method of fragmentation. Evaluation of the different precursor ion selection methods are therefore discussed using ETD LC-MS/MS data. Figure 3 shows data generated from five ETD LC-MS/MS experiments: two conducted using DDA, and three conducted using PTM-specific targeted data acquisition in which inclusion lists were generated from one preliminary LC-MS experiment. To ensure that the two precursor ion selection methods can be directly compared, the LC-MS/MS experiments were performed using replicate injections from a single methylated

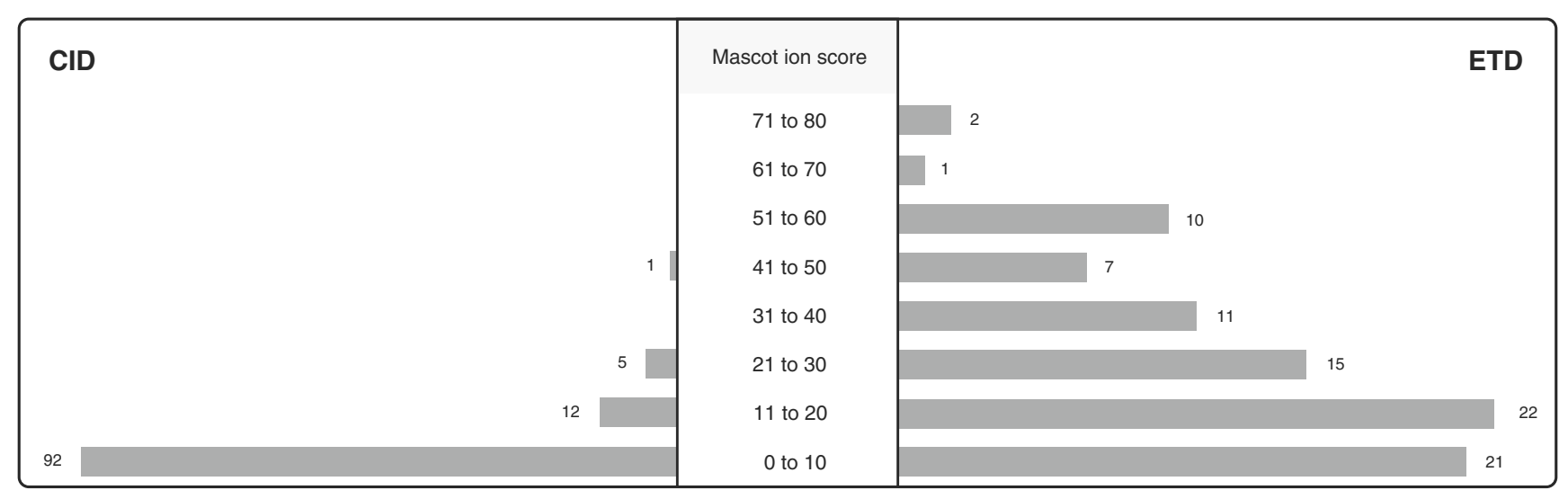

average $\mathrm{CID}$ ion score per sample $=4.3(s=5.1)$

average ETD ion score per sample $=25.2(s=1.0)$

Figure 2. Histograms illustrating Mascot ion score distributions obtained for methylated Npl3 derived peptide ions from three CID (left) and 3 ETD (right) LC-MS/MS experiments. Mascot ion scores are associated with precursor ions corresponding to the very high, high, and medium confidence methylated peptide ions in Table S-2. Searches returning incorrect peptide matches are assigned scores of 0 . Data columns are normalized to the highest value in each histogram 
in vitro methylated Npl3 peptides from purified protein digest

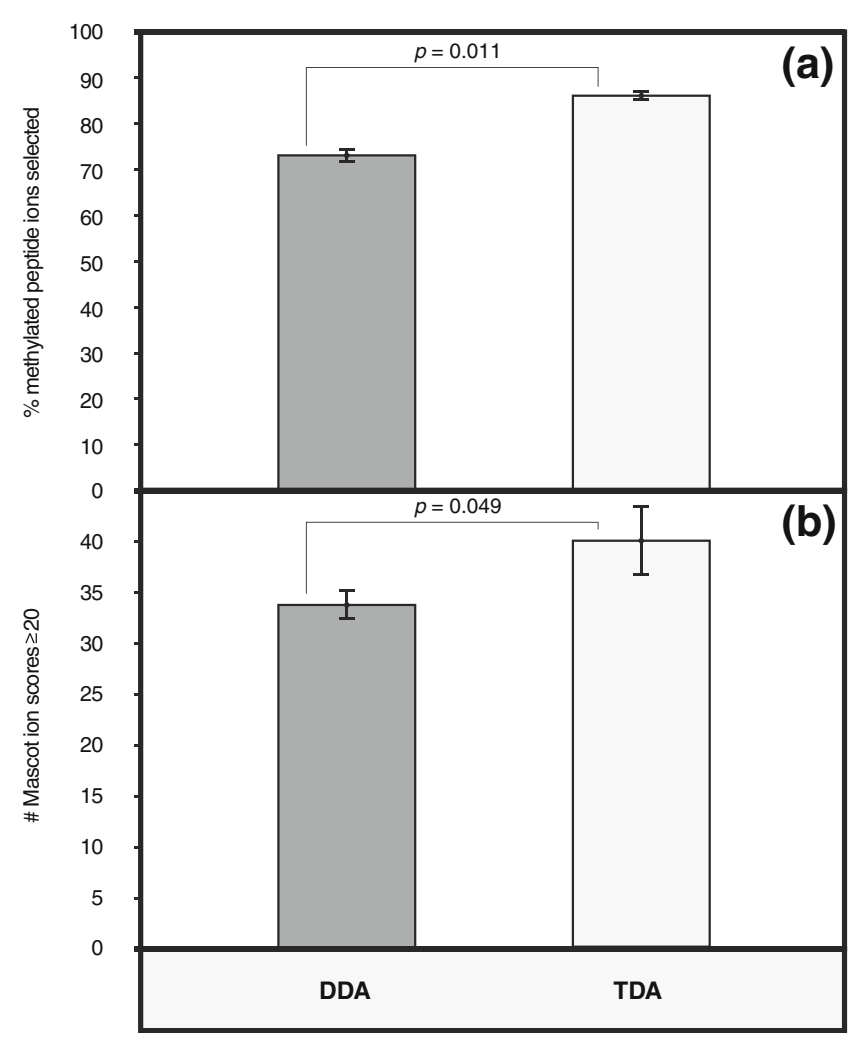

Figure 3. The (a) average percentages of methylated peptide ions selected for MS/MS, and (b) average numbers of methylated peptide ions returning Mascot ion scores $\geq 20$ from two DDA (left) and three PTM-specific targeted data acquisition (right) ETD LC-MS/MS experiments performed using replicate injections of purified and trypsin digested in vitro methylated His-Npl3. Brackets and $P$ values indicate statistical comparisons from 2-tailed $t$-tests

His-Npl3 digest sample. The data were manually inspected to ensure that the numbers of ions corresponding to the very high, high, and medium confidence methylated peptide ions of Table S-2 (based on $\mathrm{m} / z$, charge states, and retention times) observed in MS-1 are comparable across samples, and that these ions produce similar maximum ion counts (see Figure S-7). It can be seen from Figure $3 \mathrm{a}$ that the PTMspecific targeted data acquisition method reliably selects a greater proportion of methylated peptide ions than DDA; this improvement amounts to a $17 \%$ increase in the number of methylated precursor ions selected for MS/MS relative to DDA, and is statistically significant $(P=0.011$ from a 2tailed $t$-test). These findings are consistent across the very high, high, and medium confidence methylated peptide ion assignments of Table S-2 (as illustrated in Figure S-8). As a result of this improvement, $19 \%$ more methylated peptide ions (relative to DDA) are identified with Mascot ion scores $\geq 20$ when using the targeted data acquisition approach (see Figure 3b; $P=0.049$ from a 2-tailed $t$-test). Figure 4 shows histograms illustrating the maximum ion counts of the methylated peptide ions observed in the abovementioned DDA and targeted data acquisition experiments, and histograms produced using only the methylated peptide ions bypassed for fragmentation are overlaid in black. As expected, DDA performs poorly in terms of selecting low abundance methylated peptide ions for fragmentation; relative to DDA, the targeted data acquisition approach produces a $50 \%$ increase in the proportion of very low abundance peptide ions (maximum ion intensities $<6 \times 10^{4}$ cps) selected for MS/MS. This accounts for the majority of the additional ions selected when using the latter approach. However, the targeted data acquisition approach also improves the reliability of selecting higher abundance methylated peptide ions for $\mathrm{MS} / \mathrm{MS}$. Ions of maximum intensity $>1.2 \times 10^{5} \mathrm{cps}$ are, for instance, occasionally bypassed for selection during DDA, but are consistently selected using targeted data acquisition. Notably, the Mascot ion scores observed for methylated peptide ions selected using both DDA and targeted data acquisition are comparable $(25.8 \pm 1.7$ in the DDA experiments and $27.4 \pm 2.9$ in the targeted data acquisition experiments), confirming that the higher number of ion scores $\geq 20$ observed using targeted data acquisition stem from an improved ability to select methylated peptide ions for ETD.

\section{Verification on a Protein Digest Mixture Containing In Vivo Methylated Npl3}

To verify that the PTM-specific targeted data acquisition workflow remains viable when applied to a complex sample featuring peptides derived from an in vivo methylated protein, additional ETD LC-MS/MS experiments were performed. These involved six replicate injections of a protein digest mixture featuring in vivo methylated $\mathrm{Npl3}$ : three experiments were conducted using DDA, and three were conducted using PTM-specific targeted data acquisition in which inclusion lists were generated from three preliminary LC-MS experiments.

The protein identifications obtained from these experiments show that, in the sample under analysis, the in vivo methylated Npl3 peptides of interest exist within a complex mixture of peptides derived from at least 64 yeast proteins, 10 human keratin contaminants, and trypsin (as summarized in Table S-3). When considering these six experiments alone (i.e., if the experiments conducted upon the in vitro methylated His-Npl3 are not used for verification), up to 44 methylated $\mathrm{Npl} 3$ derived peptide ions can be independently identified (summarized in Table S-4): 16 precursor ions can be identified as methylated peptides with very high confidence, 25 with high confidence, and three with medium confidence according to the identification criteria defined previously. Each of these precursor ions are also identified in the in vitro methylated His-Npl3 samples, with the exception of ion \#37 of Table S-4 (the triply-charged analogue of ion \#68 of Table S-2). No novel methylation sites are found within the in vivo methylated $\mathrm{Npl} 3$ compared with the in vitro methylated His-Npl3, and 14 of the 18 methylarginine sites identified in the His-Npl3 are also found in the in vivo 
in vitro methylated Npl3 peptides from purified protein digest

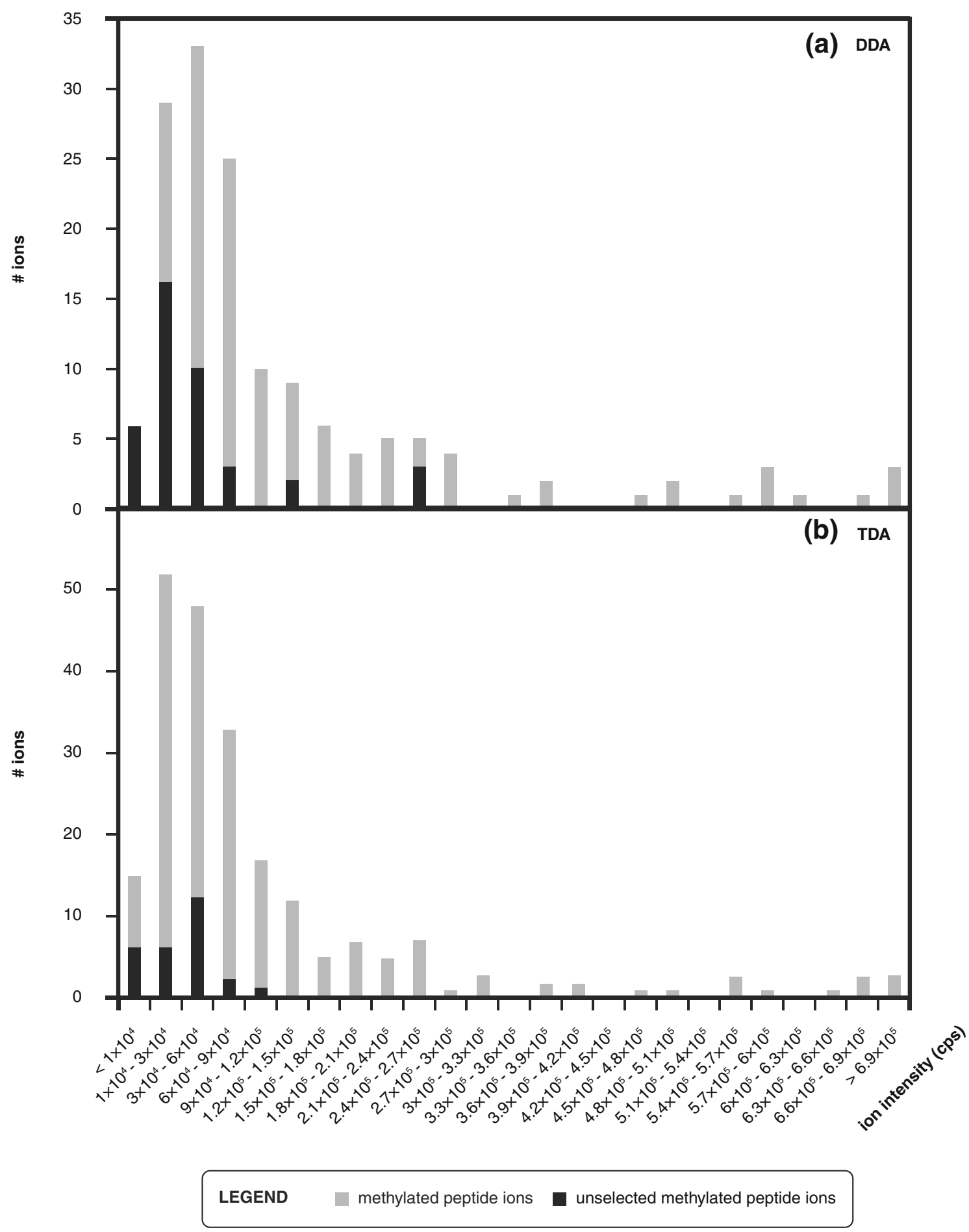

Figure 4. Histograms illustrating the maximum ion intensities for methylated peptide ions observed in the (a) two DDA and (b) three PTM-specific targeted data acquisition ETD LC-MS/MS experiments on purified and trypsin digested in vitro methylated His-Npl3 used to produce Figure 3. Total numbers of methylated peptide ions are shown in grey, and numbers of methylated peptide ions bypassed for MS/MS are shown in black

methylated protein (these sites are summarized in Scheme S-2). Each in vivo methylated residue is identified in both unmodified and (mono- and/or di-) methylated forms, confirming that the observed methylation is sub-stoichiometric.
To ensure that the different precursor ion selection methods can be directly compared, LC-MS/MS experiments were once again matched based upon the the number of methylated peptide ions able to be identified during MS-1 (based on $m / z$, 
charge states and retention times of the ions of Table S-4), and the maximum ion counts observed for these ions. Of the six LC-MS/MS experiments conducted upon the in vivo methylated Npl3 sample, two DDA and two targeted data acquisition experiments can be matched using these criteria: in each of these four experiments, every ion of Table S- 4 can be identified in MS-1 data; no significant differences in maximum ion counts are observed for these ions when these experiments are grouped according to the precursor ion selection method used (see Figure S-9). Evaluation of these four experiments reveals that the targeted data acquisition approach produces a $95 \%$ increase in the number of methylated precursor ions of Table S-4 selected for MS/MS relative to DDA, as illustrated in Figure 5a (this difference is statistically significant; $P=0.030$ from a 2-tailed $t$-test). As summarized in Figure S-10, when considering only the very high and high confidence methylated peptide ion assignments of Table S-4, the targeted data acquisition-derived improvements in methylated precursor

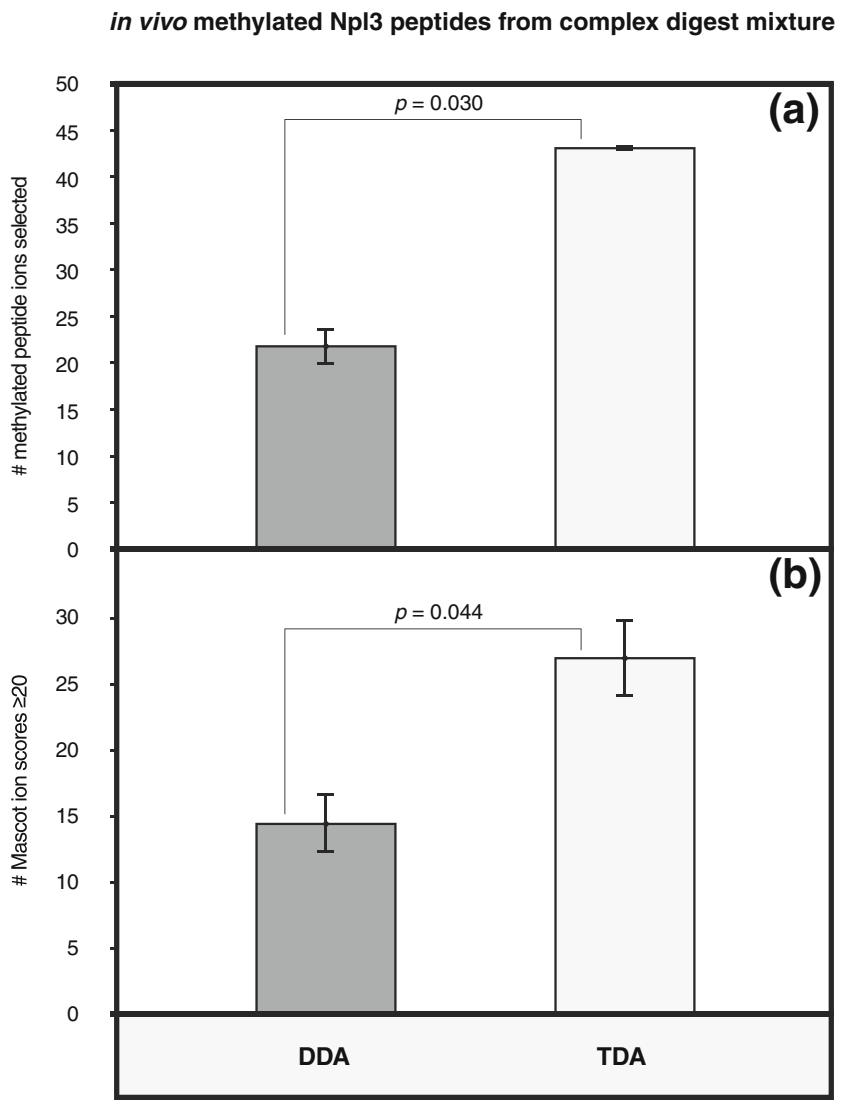

Figure 5. Results obtained from two DDA (left) and two PTM-specific targeted data acquisition (right) ETD LC-MS/ MS experiments performed using replicate injections of a single complex protein mixture featuring in vivo methylated $\mathrm{Npl}$, digested using trypsin, in terms of (a) the average number of methylated peptide ions, confidently identified from the single sample under analysis, selected for MS/MS, and (b) average numbers of these methylated peptide ions returning Mascot ion scores $\geq 20$ from. Brackets and $P$ values indicate statistical comparisons from 2-tailed $t$-tests ion selection for MS/MS remain statistically significant. When only the very high confidence methylated peptide ions are considered, a significant result is not obtained (see Figure $\mathrm{S}-10$ ); this is, however, not surprising given that the limited number of very high confidence methylated peptide ions identified from the present sample are produced at relatively high ion counts and are, therefore, less likely to be bypassed by DDA (see below). In sum, these improvements result in an $86 \%$ increase in the number of methylated peptide ions identified at Mascot ion scores $\geq 20$ (relative to DDA) when using the targeted data acquisition approach (see Figure 5b; $P=0.044$ from a 2-tailed $t$-test).

Figure 6 shows histograms illustrating the maximum ion counts of the methylated peptide ions of Table S-4 in the experiments used to produce Figure 5; ions bypassed for MS/ MS are overlaid in black. It can be seen that the DDA experiments perform particularly poorly in terms of selecting the very low abundance peptide ions (maximum ion intensities $<6 \times 10^{4} \mathrm{cps}$ ) of Table S-4 for MS/MS: $73 \%$ of such ions are bypassed for fragmentation. Higher abundance peptide ions also remain poorly selected when using DDA: $35 \%$ of the methylated peptide ions of Table S-4 with maximum ion intensities $>6 \times 10^{4} \mathrm{cps}$ are bypassed for MS/MS. In contrast, all of the ions of Table S-4 are selected for MS/MS when using the targeted data acquisition approach. It must be noted, however, that this does not imply that the targeted selection of methylated peptide ions for MS/MS is $100 \%$ comprehensive in the present experiments; as only a single in vivo methylated $\mathrm{Npl3}$ sample was analyzed, it remains likely that additional unidentified methylated peptide ions exist at low abundance within the protein digest mixture under analysis.

\section{Discussion}

The above results indicate that the PTM-specific targeted data acquisition approach outperforms DDA in both purified and complex proteins samples. Furthermore these results suggest that as sample complexity increases, the PTMspecific targeted data acquisition approach improves in performance relative to DDA. This is evidenced from a comparison of Figures $3 \mathrm{~b}$ and $5 \mathrm{~b}$; the improvements derived from the targeted data acquisition approach (relative to DDA) in the number of methylated peptide ions observed with Mascot ion scores $\geq 20$ are more pronounced in the more complex sample. The robustness of the targeted data acquisition approach to increases in sample complexity, relative to DDA, is reinforced from an inspection of Figures 4 and 6 . Inspection of parts (a) of these figures shows that whilst the maximum ion count distributions of known methylated peptide ions in the in vitro and in vivo methylated Npl3 samples remain similar, the proportion of known methylated peptide ions bypassed for fragmentation when using DDA is significantly higher in the more complex sample. In contrast, a comparison of Figures $4 \mathrm{~b}$ and $6 \mathrm{~b}$ reveals that when using the targeted data acquisition approach, no decrease in the proportion of methylated 


\section{in vivo methylated Npl3 peptides from complex digest mixture}

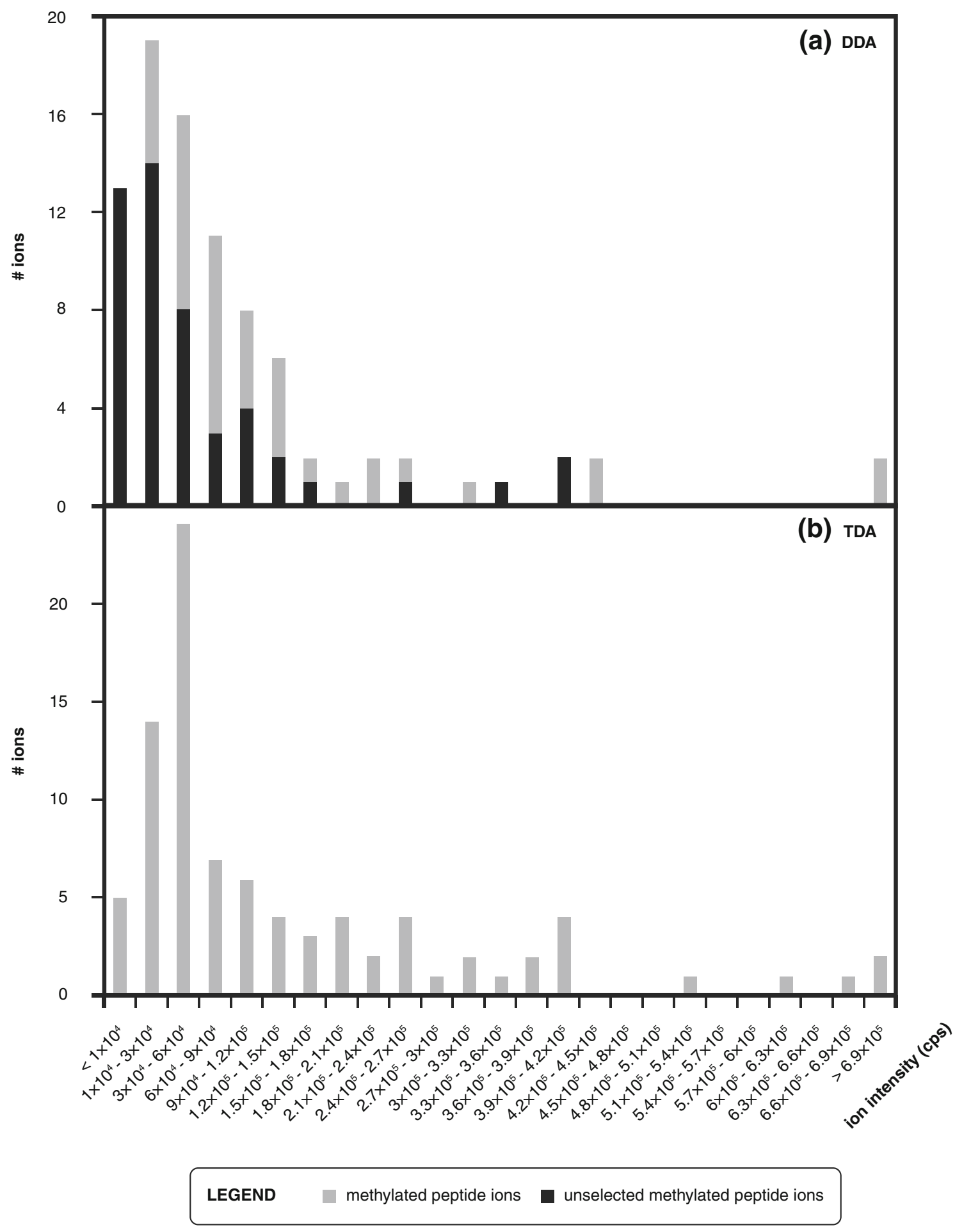

Figure 6. Histograms illustrating the maximum ion intensities for methylated peptide ions observed in the (a) two DDA and (b) two PTM-specific targeted data acquisition ETD LC-MS/MS experiments on the trypsin digested complex protein mixture featuring in vivo methylated Npl3 used to produce Figure 5. Numbers of methylated peptide ions, confidently identified from the single sample under analysis, are shown in grey, and the numbers of these methylated peptide ions bypassed for MS/MS are shown in black; additional unidentified methylated peptide ions are likely to exist in the sample under analysis

peptide ions selected for MS/MS is evident as sample complexity increases. (Figures 4 and 6 cannot, however, be compared with precision, as the data presented for the in vivo methylated $\mathrm{Np13}$ is unlikely to be comprehensive.) 
Despite the demonstrated utility of the PTM-specific targeted data acquisition approach, the analyses of the in vitro methylated His-Npl3 samples indicate that the technique nonetheless remains noncomprehensive in terms of selecting methylated peptide ions for MS/MS. In evaluating the reasons for this, the following three points should be considered: (1) the reliability of feature extraction from MS1 data (step 2 of Scheme 1); (2) the performance of step 3 of Scheme 1, in which pools of putatively methylated peptides are isolated from the extracted features; and (3) the reliability of targeted LC-MS/MS experiments in selecting the putatively methylated peptide ions for fragmentation (step 5 of Scheme 1).

In considering point (1), it is important to note that feature extraction from MS-1 data may be undertaken using a variety of different software packages (many of which have been summarized by America et al. [47]). The efficacy of this step will therefore be dependent upon the software package used. Figure S-11a illustrates the efficacy of this step for the methylated His-Npl3 sample used to produce Figures 3 and 4, analyzed across four replicate LC-MS experiments, when performed using MaxQuant. The data from each LC-MS experiment was manually inspected for the presence of any features corresponding to the very high, high, and medium confidence methylated peptide ions of Table S-2. Figure S-11a shows the average percentages of these features extracted by MaxQuant after one, two, and three experiments: $82 \% \pm 14 \%$, $88 \% \pm 7 \%$, and $92 \% \pm 4 \%$, respectively. The unextracted features are observed at relatively low maximum ion counts, as illustrated in Figure S-11b $\left(3.98 \pm 3.12 \times 10^{4}\right.$ cps unextracted and $1.28 \pm 0.20 \times 10^{5} \mathrm{cps}$ extracted; these differences are statistically significant; $P=0.020$ from a 2 -tailed $t$-test), suggesting that experimental noise may often interfere with the ability for MaxQuant to correctly recognize ${ }^{13} \mathrm{C}$ isotope distributions at low abundances. These findings account for the vast majority of the methylated peptide ions bypassed for MS/ MS when using the PTM-specific targeted data acquisition approach.

With regards to point (2), the present results indicate that inclusive but sufficiently selective pools of methylated peptides are generated during step 3 of Scheme 1 when using the FindMod utility. The different digested methylated His-Npl3 samples produce between 8500 and 23,000 features (as determined by MaxQuant). When the monoisotopic masses associated with these features are run through FindMod using a $\pm 2 \mathrm{ppm}$ mass tolerance, which is appropriate for an Orbitrap mass analyzer with a lock-mass applied [48], inclusion lists of fewer than 250 ions (typically $\sim 120$ ions) are produced from the pools of putatively methylated peptides that are returned. For the present samples, these lists contain no false negatives (the total peptide matches, false discovery and false negative rates of the FindMod analyses are elaborated upon in the Supplementary Material). Inclusion lists of this size are one order of magnitude smaller than the maximum inclusion list sizes supported by LTQ mass spectrometers [19]. With regards to point (3), the reliabilities of the present targeted LC-MS/MS experiments are, therefore, not adversely affected by mass spectrometer scan speeds. Though peptide ions targeted for MS/MS are occasionally bypassed for fragmentation when observed at extremely low ion counts ( $<3000 \mathrm{cps})$, accounting for a minor number of the missed MS/MS events for methylated peptide ions, it is unlikely for ions selected at such low abundance to produce useful MS/MS spectra.

Following consideration of points (1), (2), and (3), it can be seen that in terms of its ability to provide comprehensive selection of methylated peptide ions for MS/MS, the employed PTM-specific targeted data acquisition approach is almost exclusively limited by the noncomprehensive extraction of low abundance features following MaxQuant analysis. Despite this limitation, the approach remains readily capable of outperforming DDA after a single preliminary LC-MS experiment, as demonstrated in the data used to produce Figures 3 and 4. Furthermore, the likelihood of extracting a given methylated peptide feature is heightened over the number of preliminary LC-MS experiments conducted. If the selection of a higher proportion of methylated peptide ions than DDA is to be guaranteed, the data used to produce Figure S-11 indicates that two or more preliminary LC-MS experiments should be performed.

Comparing the present method to the targeted data acquisition approach described by Schmidt et al. [19], it is noteworthy that noncomprehensive peptide feature extraction is a limitation to both methods: if the features of PTMcarrying peptides are unable to extracted, these peptides cannot be targeted for MS/MS. Once a list of peptide features is obtained, however, the present approach provides a significantly improved means by which PTM-carrying peptides may be efficiently targeted for MS/MS. As discussed earlier, after feature extraction, only $\sim 80 \%$ of the $\sim 10,000$ extracted peptide features described by Schmidt et al. were capable of being targeted for MS/MS across five additional LC-MS/MS experiments. In contrast, the present approach allows near-comprehensive sequencing of extracted PTM-carrying peptide features of interest from similarly sized feature lists after only one additional LC-MS/ MS experiment.

\section{Conclusions}

The localization of methylarginine sites on a proteome-wide scale presents a unique set of challenges. Dense methylation of GAR domains is emerging as a common theme amongst protein arginine methyltransferase (PRMT) substrates [16, 48], and the lack of strong enrichment techniques for methylated peptides suggests that recombinant PRMT substrate production is likely to play an important role in the comprehensive localization of these sites [6, 49]. As additional PRMT substrates are identified and targeted for methylarginine site localization, the benefits of the abovementioned PTM-specific targeted data acquisition workflow should be particularly marked. 
Though demonstrated on methylarginine, the PTM-specific targeted data acquisition workflow should be equally applicable to any PTM of known mass provided the PTM-carrying peptides are observed during MS-1. Furthermore, the technique can be expanded to account for multiple different target PTMs and proteins per sample. Compared with the present study, this will involve an expansion of the search space during step 3 of Scheme 1, resulting in the generation of larger inclusion lists during step 4 of Scheme 1. The targeted LC-MS/MS techniques utilized in the current investigation are, however, readily capable of supporting inclusion lists an order of magnitude larger than those presently implemented, and the possibility exists for even larger inclusion lists to be segmented over multiple LC-MS/MS experiments.

The described PTM-specific targeted data acquisition workflow remains heavily dependent upon the observation of PTM-carrying peptides during MS-1. As such, though the approach outperforms DDA in terms of accessing low abundance PTM-carrying peptides for MS/MS, any advances in feature extraction software, and in the LC separation, ionization, or enrichment of specific PTM-carrying peptides, should enhance the utility of the approach even further.

Finally, though ETD was demonstrated to produce particularly useful fragmentation of methylarginine-carrying peptides, the PTM-specific targeted data acquisition approach is amenable to the use of alternate modes of fragmentation. The present techniques should, therefore, benefit the identification of not only methylarginine-carrying peptides but also any class of PTM-carrying peptide capable of producing useful fragmentation data from the various modes of dissociation available to contemporary MS.

\section{Acknowledgments}

The auhors thank A/Professor Mark Raftery, Dr. Ling Zhong, and Ms. Sydney Liu Lau for their maintenance of the LTQ Orbitrap Velos at the UNSW Bioanalytical Mass Spectrometry Facility. The authors also thank Dr. Elisabeth Gasteiger and Dr. Khaled Mostaguir for their maintenance of the FindMod utility (Swiss Institute of Bioinformatics). G.H.-S., M.A.E., and M.R.W. thank the Australian Research Council (ARC) for their financial support. G.H.-S. and M.A.E. also acknowledge funding from the UNSW Early Career Researcher Grants Program. J.K.K.L. acknowledges financial support from an Australian Postgraduate Award and a UNSW Research Excellence Award.

\section{References}

1. Walsh, C.T., Garneau-Tsodivoka, S., Gatto, G.J.: Protein post-translational modifications: the chemistry of proteome diversifications. Angew. Chem. 44, 7342-7272 (2005)

2. Blume-Jensen, P., Hunter, T.: Oncogenic kinase signaling. Nature 411, 355-365 (2001)
3. Hunter, T.: Protein kinases and phosphatases: the yin and yang of protein phosphorylation and signalling. Cell 80, 225-236 (1995)

4. Huang, J., Berger, S.L.: The emerging field of dynamic lysine methylation of non-histone proteins. Curr. Opin. Genet. Dev. 18, 152-158 (2008)

5. Bedford, M.T., Clarke, S.: Protein arginine methylation in mammals: who, what, and why. Mol. Cell 33, 1-13 (2009)

6. Erce, M.A., Pang, C.N.I., Hart-Smith, G., Wilkins, M.R.: The methylproteome and the intracellular methylation network. Proteomics, in press (2012)

7. Bedford, M.T., Richard, S.: Arginine methylation: an emerging regulator of protein function. Mol. Cell 18, 263-272 (2005)

8. Piak, W.K., Piak, D.C., Kim, S.: Historical review: the field of protein methylation. Trends Biochem. Sci. 32, 146-152 (2007)

9. Witze, E.S., Old, W.M., Resing, K.A., Ahn, N.G.: Mapping protein post-translational modifications with mass spectrometry. Nat. Methods 4, 798-806 (2007)

10. de Godoy, L.M.F., Olsen, J.V., de Souza, G.A., Li, G.Q., Mortensen, P., Mann, M.: Status of complete proteome analysis by mass spectrometry: SILAC labeled yeast as a model system. Genome Biology 7, R50 (2006)

11. Picotti, P., Aebersold, R., Domon, B.: The implications of proteolytic background for shotgun proteomics. Mol. Cell. Proteom. 6, 1589-1598 (2007)

12. Brunner, E., Ahrens, C.H., Mohanty, S., Baetschmann, H., Loevenich, S., Potthast, F., Deutsch, E.W., Panse, C., de Lichtenberg, U., Rinner, O., Lee, H., Pedroli, P.G.A., Malmstrom, J., Koehler, K., Schrimpf, S., Krijgsveld, J., Kregenow, F., Heck, A.J.R., Hafen, E., Schlapbach, R., Aebersold, R.: A high-quality catalogue of the Drosphila melanogaster proteome. Nat. Biotechnol. 25, 576-583 (2007)

13. Trost, M., Bridon, G., Desjardins, M., Thibault, P.: Subcellular phosphoproteomics. Mass Spectrom. Rev. 29, 962-990 (2010)

14. Sarma, K., Nishioka, K., Reinberg, D. In Methods Enzymol., Allis, C. D.; Carl, W., Eds. Academic Press: 2003; pp 255-269

15. Perez-Burgos, L., Peters, A. H. F. M., Opravil, S., Kauer, M., Mechtler, K., Jenuwein, T. In Methods Enzymol., Allis, C. D.; Carl, W., Eds. Academic Press: 2003; pp 234-254

16. Ong, S.E., Mittler, G., Mann, M.: Identifying and quantifying in vivo methylation sites by heavy methyl SILAC. Nat. Methods 1, 1-8 (2004)

17. Calvo, S., Jain, M., Xie, X., Sheth, S.A., Chang, B., Goldberger, O.A., Spinazzola, A., Zeviani, M., Carr, S.A., Mootha, V.K.: Systematic identification of human mitochondrial disease genes through integrative genomics. Nat. Genet. 38, 576-582 (2006)

18. Savitski, M.M., Fischer, F., Mathieson, T., Sweetman, G., Lang, M., Bantscheff, M.: Targeted data acquisition for improved reproducibility and robustness of proteomic mass spectrometry assays. J. Am. Soc. Mass Spectrom. 21, 1668-1679 (2010)

19. Schmidt, A., Gehlenborg, N., Bodenmiller, B., Mueller, L.N., Campbell, D., Mueller, M., Aebersold, R., Domon, B.: An integrated, directed mass spectrometric approach for in-depth characterization of complex peptide mixtures. Mol. Cell. Proteom. 7, 2138-2150 (2008)

20. Domon, B., Bodenmiller, B., Carapito, C., Hao, Z., Huehmer, A., Aebersold, R.: Electron transfer dissociation in conjunction with collision activation to investigate the Drosophila melanogaster phosphoproteome. J. Proteome Res. 8, 2633-2639 (2009)

21. Michalski, A., Cox, J., Mann, M.: More than 100,000 detectable peptide species elute in single shotgun proteomics runs but the majority is inaccessible to data-dependent LC-MS/MS. J. Proteome Res. 10, 1785-1793 (2011)

22. Syka, J.E.P., Coon, J.J., Schroeder, M.J., Shabanowitz, J., Hunt, D.F.: Peptide and protein sequence analysis by electron trasnsfer dissociation mass spectrometry. PNAS 101, 9528-9533 (2004)

23. Coon, J.J., Shabanowitz, J., Hunt, D.F., Syka, J.E.P.: Electron transfer dissociation of peptide anions. J. Am. Soc. Mass Spectrom. 16, 880-882 (2005)

24. Mikesh, L.M., Ueberheide, B., Chi, A., Coon, J.J., Syka, J.E.P., Shabanowitz, J., Hunt, D.F.: The utility of ETD mass spectrometry in proteomic analysis. Biochim. Biophys. Acta: Proteins Proteomics 1764, 1811-1822 (2006)

25. Gehrig, P.M., Hunziker, P.E.: Fragmentation pathways of $\mathrm{N}^{\mathrm{G}}$ methylated and unmodified arginine residues in peptides studied by ESI-MS/MS and MALDI-MS. J. Am. Soc. Mass Spectrom. 15, 142-149 (2004)

26. Brame, C.J., Moran, M.F., McBroom-Cerajewski, L.D.B.: A mass spectrometry based method for distinguishing between symmetrically 
and asymmetrically dimethylated arginine residues. Rapid Commun. Mass Spectrom. 18, 877-881 (2004)

27. Wang, H., Straubinger, R.M., Aletta, J.M., Cao, J., Duan, X., Yu, H., $\mathrm{Qu}, \mathrm{J} .:$ Accurate localization and relative quantification of arginine methylation using nanoflow liquid chromatography coupled to electron transfer dissociation and orbitrap mass spectrometry. J. Am. Soc. Mass Spectrom. 20, 507-519 (2009)

28. Snijders, A.P.L., Hung, M.L., Wilson, S.A., Dickman, M.J.: Analysis of arginine and lysine methylation utilizing peptide separations at neutral $\mathrm{pH}$ and electron transfer dissociation mass spectrometry. J. Am. Soc. Mass Spectrom. 21, 88-96 (2010)

29. Henry, M.F., Silver, P.A.: A novel methyltransferase (Hmtlp) modifies poly(a)+-RNA-binding proteins. Mol. Cell. Biol. 16, 3668-3678 (1996)

30. Siebel, C.W., Guthrie, C.: The essential yeast RNA binding protein Np13p is methylated. PNAS 93, 13641-13646 (1996)

31. McBride, A.E., Weiss, V.H., Kim, H.K., Hogle, J.M., Silver, P.A.: Analysis of the yeast arginine methyltransferase Hmtlp/Rmtlp and its in vivo function. J. Biol. Chem. 275, 3128-3136 (2000)

32. McBride, A.E., Cook, J.T., Stemmler, E.A., Rutledge, K.L., McGrath, K.A., Rubens, J.A.: Arginine methylation of yeast mRNA-binding protein $\mathrm{Npl3}$ directly affects its function, nuclear export, and intranuclear protein interactions. J. Biol. Chem. 280, 30888-30898 (2005)

33. Yu, M.C., Bachand, F., McBride, A.E., Komili, S., Casolari, J.M., Silver, P.A.: Arginine methyltransferase affects interactions and recruitment of mRNA processing and export factors. Genes Dev. 18, 2024-2035 (2004)

34. Shen, Y.F., Henry, M.F., Weiss, V.H., Valentini, S.R., Silver, P.A., Lee, M.S.: Arginine methylation facilitates the nuclear export of hnRNP proteins. Genes Dev. 12, 679-691 (1998)

35. Shevchenko, A., Wilm, M., Vorm, O., Mann, M.: Mass spectrometric sequencing of proteins from silver-stained polyacrylamide gels. Anal. Chem. 68, 850-858 (1996)

36. Chiu, J., Tillet, D., Dawes, I.W., March, P.E.: Site-directed, Ligaseindependent mutagenesis (SLIM) for highly efficient mutagenesis of plasmids greater than $8 \mathrm{~kb}$. J. Microbiol. Methods 73, 195-198 (2008)

37. Gietz, R.D., Schiestl, R.H., Willems, A.R., Woods, R.A.: Studies on the transformation of intact yeast cells by the LiAc/SS-DNA/PEG procedure. Yeast 11, 355-360 (1995)

38. Gelperin, D.M., White, M.A., Wilkinson, M.L., Kon, Y., Kung, L.A., Wise, K.J., Lopez-Hoyo, N., Jiang, L.X., Piccirillo, S., Yu, H.Y., Gerstein, M., Dumont, M.E., Phizicky, E.M., Snyder, M., Grayhack,
E.J.: Biochemical and genetic analysis of the yeast proteome with a movable ORF collection. Genes Dev. 19, 2816-2826 (2005)

39. Mok, J., Im, H., Snyder, M.: Global identification of protein kinase substrates by protein microarray analysis. Nat. Protoc. 4, 1820-1827 (2009)

40. Hart-Smith, G., Raftery, M.J.: Detection and characterization of low abundance glycopeptides via higher-energy c-trap dissociation and orbitrap mass analysis. J. Am. Soc. Mass Spectrom. 23, 124-140 (2012)

41. Cox, J., Mann, M.: MaxQuant enables high peptide identification rates, individualized ppb-range mass accuracies and proteome-wide protein quantification. Nat. Biotechnol. 26, 1367-1372 (2008)

42. Wilkins, M.R., Gasteiger, E., Gooley, A.A., Herbert, B.R., Molloy, M.P., Binz, P.A., Ou, K., Sanchez, J.C., Bairoch, A., Williams, K.L., Hochstrasser, D.F.: High-throughput mass spectrometric discovery of protein post-translational modifications. J. Mol. Biol. 289, 654-657 (1999)

43. Chen, Y.-C., Milliman, E.J., Goulet, I., Côté, J., Jackson, C.A., Vollbracht, J.A., Yu, M.C.: Protein arginine methylation facilitates cotranscriptional recruitment of pre-mRNA splicing factors. Mol. Cell. Biol. 30, 5245-5256 (2010)

44. Rappsilber, J., Friesen, W.J., Paushkin, S., Dreyfuss, G., Mann, M.: Detection of arginine dimethylated peptides by parallel precursor ion scanning mass spectrometry in positive ion mode. Anal. Chem. 75, 3107-3114 (2003)

45. McAlister, G.C., Phanstiel, D., Good, D.M., Berggren, T., Coon, J.J.: Implementation of electron-transfer dissociation on a hybrid linear ion trap-orbitrap mass spectrometer. Anal. Chem. 79, 3525-3534 (2007)

46. Frese, C.K., Altelaar, A.F., Hennrich, M.L., Nolting, D., Zeller, M., Griep-Raming, J., Heck, A.J., Mohammed, S.: Improved peptide identification by targeted fragmentation using CID, HCD, and ETD on an LTQ-Orbitrap Velos. J. Proteome Res. 10, 2377-2388 (2011)

47. America, A.H.P., Cordewener, J.H.G.: Comparative LC-MS: A landscape of peaks and valleys. Proteomics 8, 731-749 (2008)

48. Boisvert, F.M., Cote, J., Boulanger, M.C., Richard, S.: A proteomic analysis of arginine-methylated protein complexes. Mol. Cell. Proteom. 2, 1319-1330 (2003)

49. Hsieh, C.H., Huang, S.Y., Wu, Y.C., Liu, L.F., Han, C.C., Liu, Y.C., Tam, M.F.: Expression of proteins with dimethylarginines in Escherichia coli for protein-protein interaction studies. Protein Sci. 16, 919928 (2007) 